J. Dairy Sci. 90:2579

doi:10.3168/jds.2007-0127

(c) American Dairy Science Association, 2007.

\title{
Letter to the Editor: Mastitis Treatment Trials
}

\section{S. Pyörälä}

University of Helsinki, Faculty of Veterinary Medicine, Department of Production Animal Medicine, FI-04920 Saarentaus, Finland

Published articles on antimicrobial treatment of mastitis often lack some important information. These are details of the pharmacokinetics and pharmacodynamics of the antimicrobial agents used, including the in vitro susceptibility of the bacteria isolated from the treated cows to the drug used in the treatment. The isolated bacterial species should be identified to the species level and results for each species presented separately; it is not adequate to pool bacteria into groups such as "environmental streptococci" or "coliforms." The possible effect of different antimicrobial agents on resistance development should also be considered, at least briefly. Emergence of resistance can develop among mastitis pathogens, but also among other bacteria; for example, in the so-called commensals. Some antimicrobial agents

Received February 20, 2007.

Accepted April 3, 2007.

E-mail: Satu.pyorala@helsinki.fi may induce this development more easily than others. The parenteral route of administration is related to a significantly higher risk of selecting for resistance compared with intramammary administration. Treatment recommendations should take this aspect into account. Information about the achieved and maintained drug levels in the milk, preferably in relation to the MIC values of the target pathogens, would be of interest to readers. Thus, for parenteral treatments, dosing should be expressed in milligrams per kilogram and not, for example, in grams per animal, which is inaccurate because the BW of the dairy cows may vary widely. All of this information would help to conclude the possible efficacy and usefulness of the treatments reported in the study. Long descriptions of the statistical methods do not help if the aspects mentioned above are missing. The mastitis treatment studies reported in the Journal of Dairy Science should meet the scientific criteria of high-quality treatment studies. 